\title{
Investigating the origin of the spectral line profiles of the Hot Wolf-Rayet Star WR 2
}

A.-N. Chené ${ }^{\oplus},{ }^{1,2,3 \star}$ N. St-Louis ${ }^{\oplus},{ }^{4}$ A. F. J. Moffat, ${ }^{4}$ O. Schnurr, ${ }^{5}$ P. A. Crowther, ${ }^{6}$ G. A. Wade, ${ }^{7}$ N. D. Richardson ${ }^{\odot},{ }^{8}$ C. Baranec, ${ }^{9}$ C. A. Ziegler, ${ }^{10}$ N. M. Law, ${ }^{10}$ R. Riddle ${ }^{\odot},{ }^{11}$ G. A. Rate, ${ }^{6}$ É. Artigau ${ }^{4}$ and E. Alecian ${ }^{12}$ BinaMIcS collaboration

${ }^{1}$ Gemini Observatory, Northern Operations Center, 670 North A'ohoku Place, Hilo, HI 96720, USA

${ }^{2}$ Departamento de Física y Astronomía, Universidad de Valparaíso, Av. Gran Bretaña 1111, Playa Ancha, Casilla 5030, Chile

${ }^{3}$ Departamento de Astronomía, Universidad de Concepción, Casilla 160-C, Chile

${ }^{4}$ Département de Physique, Université de Montréal, C. P. 6128, succ. centre-ville, Montréal (Qc) H3C $3 J 7$ Centre de Recherche en Astrophysique du Québec, Canada

${ }^{5}$ Leibniz-Institut für Astrophysik Potsdam (AIP), An der Sternwarte 16, D-14482 Potsdam, Germany

${ }^{6}$ Department of Physics and Astronomy, University of Sheffield, Sheffield S3 7RH, UK

${ }^{7}$ Department of Physics, Royal Military College of Canada, PO Box 17000, Stn Forces, Kingston, K7K 7B4, Canada

${ }^{8}$ Ritter Observatory, Department of Physics and Astronomy, The University of Toledo, Toledo, OH 43606-3390, USA

${ }^{9}$ Institute for Astronomy, University of Hawai' 'i at Mānoa, Hilo, HI 96720-2700, USA

${ }^{10}$ Dunlap Institute for Astronomy and Astrophysics, University of Toronto, Ontario M5S 3H4, Canada

${ }^{11}$ Division of Physics, MathematicsAstronomy, California Institute of Technology, Pasadena, CA 91125, USA

${ }^{12}$ Université Grenoble Alpes, CNRS, IPAG, F-38000 Grenoble, France

\begin{abstract}
The hot WN star WR 2 (HD 6327) has been claimed to have many singular characteristics. To explain its unusually rounded and relatively weak emission line profiles, it has been proposed that WR 2 is rotating close to break-up with a magnetically confined wind. Alternatively, the line profiles could be explained by the dilution of WR 2's spectrum by that of a companion. In this paper, we present a study of WR 2 using near-infrared AO imaging and optical spectroscopy and polarimetry. Our spectra reveal the presence of weak photospheric absorption lines from a B 2.5-4V companion, which however contributes only 5-10 percent to the total light, suggesting that the companion is a background object. Therefore, its flux cannot be causing any significant dilution of the WR star's emission lines. The absence of intrinsic linear continuum polarization from WR 2 does not support the proposed fast rotation. Our Stokes V spectrum was not of sufficient quality to test the presence of a moderately strong organized magnetic field but our new modelling indicates that to confine the wind the putative magnetic field must be significantly stronger than was previously suggested sufficiently strong as to make its presence implausible.
\end{abstract}

Key words: stars: individual: WR 2 -stars: magnetic fields-stars: rotation-stars: winds, outflows - stars: Wolf-Rayet.

\section{INTRODUCTION}

Classical Wolf-Rayet (WR) stars are the chemically evolved descendants of $\mathrm{O}$ stars, possessing a unique spectroscopic signature of strong, broad emission lines, arising within their fast, dense winds (Crowther 2007). Their two main subclasses, WN and WC, reflect the products of core hydrogen and helium burning, respectively. The

^E-mail: achene@gemini.edu former is commonly subdivided into early (WN2-6) and late (WN711) types, depending upon the degree of ionization of helium and nitrogen (Smith, Shara \& Moffat 1996). Early-type WN stars in the Milky Way exhibit either strong, broad Gaussian line profiles (e.g. WR6, WN4b), or weak, narrow triangular profiles [e.g. WR152, WN3(h)], with one notable exception: WR 2 (WN2b), the subject of this study.

WR 2 (HD 6327) is the only known WN2 star in our Galaxy. Its spectrum displays relatively weak 'bowler-hat'-shaped broad 
emission lines, in stark contrast with the more normal strong (Gaussian) or weak (triangular) profiles of other early-type WN stars. Considering that WR 2 has an extreme combination of a small radius, fairly low mass-loss rate and high temperature (Hamann, Gräfener \& Liermann 2006), a certain line dilution due to the Baldwin effect (van Gent et al. 2001) is expected. However, as we will present below, this does not seem to be sufficient to explain the line profiles.

Two scenarios have been proposed to explain this unusual characteristic. The first involves a companion star, where intrinsically strong Gaussian lines appear weak owing to significant line dilution from a companion (Crowther 1993). However, convincing evidence of such a companion has not been reported in the literature to date. The second scenario involves the intrinsic Gaussian/triangular lines being modified by rapid rotation. The Potsdam group has analysed WR 2 using the latest version of their model atmosphere code (Hamann et al. 2006), and remarkably the model spectrum failed to reproduce its line profiles, unless it was folded with a rotation profile near break-up velocity, with an equatorial rotation speed of $1900 \mathrm{~km}$ $\mathrm{s}^{-1}$. Of course, as mentioned by the authors themselves, it is a purely mathematical solution, as a mere solid rotation is not physical and many complex interactions are expected when such a fast rotation speed occurs. More recently, Shenar, Hamann \& Todt (2014) have developed a more detailed atmospheric model which proposes a much slower, and realistic surface rotation speed $\left(200 \mathrm{~km} \mathrm{~s}^{-1}\right)$, but a very strong magnetic field of $40 \mathrm{kG}$ at the surface to force rigid rotation of the wind up to a radius $10 R_{*}$.

Four more 'round-lined' WR stars are known in the LMC, BAT99 7, 51, 88, and 94 (Rühling 2008; Shenar et al. 2014), but this type of line profile still remains a very rare oddity. As WR 2 has an apparent magnitude that is at least 4 mag brighter than the more distant LMC stars, it is a perfect proxy to study the phenomenon.

If WR 2's claimed rapid rotation were confirmed, it would make it a prime long-soft gamma-ray burst (LGRB) progenitor candidate. LGRBs are currently best explained by the "collapsar' model (Woosley 1993; MacFadyen \& Woosley 1999; MacFadyen, Woosley \& Heger 2001), involving a rapidly rotating, fully hydrogen-depleted WR star undergoing core collapse. Classical WR stars are the helium-burning cores of evolved massive stars in their last visible evolutionary phase before they explode as type $\mathrm{Ib} / \mathrm{c}$ core-collapse supernovae (ccSNe). Direct observational evidence of a type-Ic-LGRB connection (Kelly, Kirshner \& Pahre 2008) lends considerable weight to the collapsar model, and might imply that LGRB progenitors are merely the rapidly rotating tail in the distribution of type Ic progenitors. The mere presence of rapid rotation can in principle be revealed quite easily by linear spectropolarimetry (Harries 2000): the rotation causes axisymmetric distortions of stellar winds, which in turns causes polarization from scattered radiation, and since the continuum radiation is formed at smaller radii than the line emission, the net effect is an apparent decrease of polarization in the lines.

On the other hand, if the proposed strong magnetic field of WR 2 was confirmed, it would make it a prime magnetar progenitor candidate. Magnetars are neutron stars with surface magnetic fields as strong as $10^{14}-10^{15} \mathrm{G}$ (e.g. Ferrario \& Wickramasinghe 2006). The current explanation for the origin of a neutron star's magnetic field is the conservation of the star's magnetic flux during core collapse; magnetars could then be descendants of the most strongly magnetized WR stars (Gaensler et al. 2005). The shape of the WR wind would also be affected by its confinement by the strong magnetic field (Owocki \& ud-Doula 2004; Townsend \& Owocki
2005; ud-Doula et al. 2013), and its asymmetry could be detected in linear spectropolarimetry.

This study presents our investigation of the origin of the rounded emission line profiles of WR 2. In Section 2, we search for any manifestation of a companion star and investigate how its presence can lead to round emission lines. We search for evidence of rapid stellar rotation in Section 3, where we present new linear broadband polarimetry as well as spectropolarimetry to assess the symmetry of WR 2's wind. We look for evidence of the presence of a strong magnetic field in Section 4, where we discuss our attempt to detect a global magnetic field in WR 2 using the Zeeman effect (Section 4.1). Finally, all our results are summarized in Section 5, where we present what we have learned from these observations about the true nature of WR 2 .

\section{SEARCH FOR A COMPANION STAR}

Before testing any extreme stellar parameter of WR 2, we first verify the possibility that its lines are diluted by the flux of a companion.

\subsection{Observations}

\subsubsection{Spectroscopy}

This study is based on a series of published and unpublished, archival and new spectra from different observatories. This includes spectra from the $1.6 \mathrm{~m}$ telescope of the Observatoire du Mont Mégantic (OMM), the 4.2m William Herschel Telescope (WHT) and the $1.85 \mathrm{~m}$ telescope of the Dominion Astrophysical Observatory (DAO) obtained over nearly $10 \mathrm{yr}$. We add to that a highresolution spectrum obtained with the ESPaDOnS spectropolarimeter at the 3.6m Canada-France-Hawaii Telescope (CFHT) that we present in more detail in Section 3.2, and high $\mathrm{S} / \mathrm{N}$ spectra obtained using GMOS at the $8.2 \mathrm{~m}$ Gemini-North telescope (originally obtained to search for line profile variations). Finally, we also include $2.5 \mathrm{~m}$ Isaac Newton Telescope (INT) spectrophotometry which is used for modelling in Section 2.4. When series of spectra were obtained, all were combined into nightly averages to optimize the $\mathrm{S} / \mathrm{N}$. The details of the spectroscopic observations are summarized in Table 1 .

\subsubsection{Direct imaging}

In an effort to spatially isolate a putative companion of WR 2, we first acquired visible-light adaptive optics imaging of WR 2 using the Robo-AO system (Baranec et al. 2014) on the 1.5m Telescope at Palomar Observatory on 2014 November 9. The observation in the Sloan $i$ band comprises a sequence of full-frame-transfer detector readouts at the maximum rate of $8.6 \mathrm{~Hz}$ for a total of $120 \mathrm{~s}$ of integration time. The individual images were then combined using post-facto shift-and-add processing using WR 2 as the tip-tilt star with 100 per cent frame selection (Law et al. 2014).

We also obtained time for a Poor Weather program at GeminiNorth to observe a diffraction-limited image with NIRI + Altair in the $K$ band. Those data required basic data reduction processes using the Gemini IRAF ${ }^{1}$ packages for NIRI.

\footnotetext{
${ }^{1}$ IRAF is distributed by the National Optical Astronomy Observatories (NOAO), which is operated by the Association of Universities for Research in Astronomy, Inc. (AURA) under cooperative agreement with the National Science Foundation (NSF).
} 
Table 1. Spectroscopic data.

\begin{tabular}{|c|c|c|c|c|c|c|c|c|c|}
\hline $\begin{array}{l}\text { Telescope } \\
\text { instrument }\end{array}$ & Dates & $\begin{array}{c}\lambda \text { Coverage } \\
\AA\end{array}$ & $\begin{array}{c}\Delta \lambda \\
\text { (3 pixels) }\end{array}$ & $\mathrm{S} / \mathrm{N}$ & $\begin{array}{l}\text { Telescope } \\
\text { instrument }\end{array}$ & Dates & $\begin{array}{c}\lambda \text { Coverage } \\
\AA\end{array}$ & $\begin{array}{c}\Delta \lambda \\
\text { (3 pixels) }\end{array}$ & $\mathrm{S} / \mathrm{N}$ \\
\hline INT/IDS & 1991 Sep 19-22 & $3800-7290$ & $2-2.7 \AA$ & 30 & $\mathrm{DAO}^{a}$ & 2008 Aug 15 & $4300-5090$ & $2.3 \AA$ & 340 \\
\hline \multirow[t]{2}{*}{$\mathrm{OMM}^{\mathrm{b}}$} & 2002 Jul 21, 25; & $4500-5200$ & $1.6 \AA$ & 100 & $\mathrm{CFHT}^{\mathrm{c}}$ & 2010 Aug 03 & $3700-10480$ & $0.06 \AA$ & 100 \\
\hline & 2002 Oct $19-23$ & & & & Gemini/ & 2012 Jul 11-23 & $3970-5435$ & $1.5 \AA$ & 500 \\
\hline WHT/ISIS & 2002 Nov 19 & $4245-5130$ & $0.6 \AA$ & 200 & GMOS $^{\mathrm{c}}$ & Aug 31 & & & \\
\hline
\end{tabular}

Notes: ${ }^{a}$ Published by Chené, Moffat \& Crowther (2008).

${ }^{b}$ Published by St-Louis et al. (2009).

${ }^{\mathrm{c}}$ This study.

\subsection{Search for a companion spectrum}

An inspection of the WR 2 spectrum reveals clear narrow absorption lines mostly associated with $\mathrm{H}$ and $\mathrm{He} \mathrm{I}$ in all our spectra. To identify the spectral lines, we have averaged all GMOS spectra to reach a $\mathrm{S} / \mathrm{N} \sim 1300$ per resolution element in the continuum. The result is presented for the region 4050-4570 ̊ in Fig. 1, where the absorption features from a companion and those formed in the interstellar medium (ISM) are marked and identified. A few more absorption lines are visible in the rest of the spectra, and are mostly associated to $\mathrm{H}$ or are interstellar (IS) lines. The equivalent width ratio of the He I $\lambda 4471$ to the $\mathrm{Mg}$ II $\lambda 4481$ lines is 1.95 , which corresponds to a spectral type of B 2.5-4 (a B 2.5 type will be used from now on for convenience) and the ratio of the $S$ III $\lambda 4552$ (barely visible in our spectrum) to the He I $\lambda 4387$ line suggests a luminosity class of V (Walborn \& Fitzpatrick 1990). The very faint Si II $\lambda 4128,30$ and C II $\lambda 4267$ lines can barely be distinguished.

The closest known star to WR 2 TYC4017-1829-2, has a $V=$ $14.81 \mathrm{mag}$ (compared to $V=10.99 \mathrm{mag}$ for WR2) and is separated by 13.8 arcsec. This is typically 5 times the size of the seeing disc at the smaller telescopes used for this study, and more than 10 times the size of the seeing disc at the Maunakea Observatories. Hence, conclude that there is no significant contamination of WR 2's spectrum by this star, and the absorption lines likely come from a companion to WR 2 .

\subsection{Search for radial velocity variations}

In order to determine if the B companion is bound to WR 2 or is an accidentally aligned star at a different distance, we first searched for radial velocity (RV) changes in both the emission and newly discovered absorption lines. We used the many IS lines across the spectrum as references for RV, which allows one to correct for zero-point shifts in spectra coming from various instruments. The search for RV variations of the WR star was done using crosscorrelation of the spectra in the region from $4300 \AA$ to $5000 \AA$, with the GMOS spectrum from the night of 2012 August 31 as reference. The signal of the cross-correlation profile is dominated by the WR emission lines. The error on the values is given by the width of the cross-correlation profile, which typically varies between 10 and $30 \mathrm{~km} \mathrm{~s}^{-1}$. Those errors remain small regardless of the broadness of the WR lines due to the wide wavelength coverage of the spectra. The result is presented in Fig. 2, using black diamond symbols. The amplitude of the measured RV variation is very small, and when it is compared to the $1 \sigma$ error bars, we see that the variations rarely exceed the $1 \sigma$ or $2 \sigma$ level. The RV of the absorption line star was obtained by fitting the absorption lines with a Voigt profile. For clarity, we assume that the lines of a given element move together, hence we plotted only an average value for the $\mathrm{H}$ (red triangles) and $\mathrm{He}$ (red squares) lines. The RV values for the $\mathrm{H}$ and $\mathrm{He}$ lines were corrected by subtracting their time-median value, so they can be compared with the WR RV values centred on $0 \mathrm{~km} \mathrm{~s}^{-1}$. The error on the values depends highly on the spectra signal-to-noise ratio, since the absorption lines are shallow, and in fairly small number per specie. Once again, when compared with the error bars, the variations barely exceed $1 \sigma$ (except in few rare cases when the emission and absorption lines seem to have fortuitously moved together). We therefore conclude that we do not detect any significant RV motion of the two components, and we need another test to determine if the B star and WR 2 form a wide binary system or not.

\subsection{Flux ratio measurement from direct imaging}

Using high-resolution imaging, we successfully detected a faint source at a separation of $0.25 \pm 0.06$ and a position angle of $296 \pm 5^{\circ}$ with respect to WR 2 (see Fig. 3). No other star is visible down to a radius of 0.05 arcsec (i.e. half the seeing disc FWHM) around WR 2. Based on the flux ratio and close separation, we suspect that the detected source is the B star whose line we detected in our spectrum .

Using multiple aperture photometry on the Robo-AO data and subtraction of the primary point spread function, we measure a contrast of $2.4 \pm 0.3$ mag in i' (corresponding to a factor 9 difference in flux). NIRI observations reveal a visual companion that is a factor of 16 fainter (a 3 mag difference), located 0.25 arcsec away to the North West from WR 2 (compatible with the previously mentioned PA). Of particular interest is this NIR flux ratio. Our spectroscopy shows evidence that the B star contributes about 5 percent of the flux in the optical, consistent with the 7 percent seen here in the NIR (see next section). Despite the extreme luminosity differences, the B star should be almost as bright in the optical as in the NIR if it were at the same distance as WR 2. As this is clearly not the case, we suggest that the B star is a background star with a slightly higher reddening than that of WR 2 .

\subsection{Contribution of the B star}

WR 2 is believed to be a member of the Cas OB1 association. Although WR 2 does not have a direct parallax from Gaia DR2, we can infer its distance using the parallaxes of eight other association members (noted by Mel'nik \& Dambis 2017). Two further members were excluded due to their abnormally high and low parallaxes (approximately 0.8 mas and 0.04 mas, respectively) and the remaining member was not observed in Gaia. The proper motions of the members support the conclusion that Cas OB1 is a viable association. Using the distance calculation methods of 


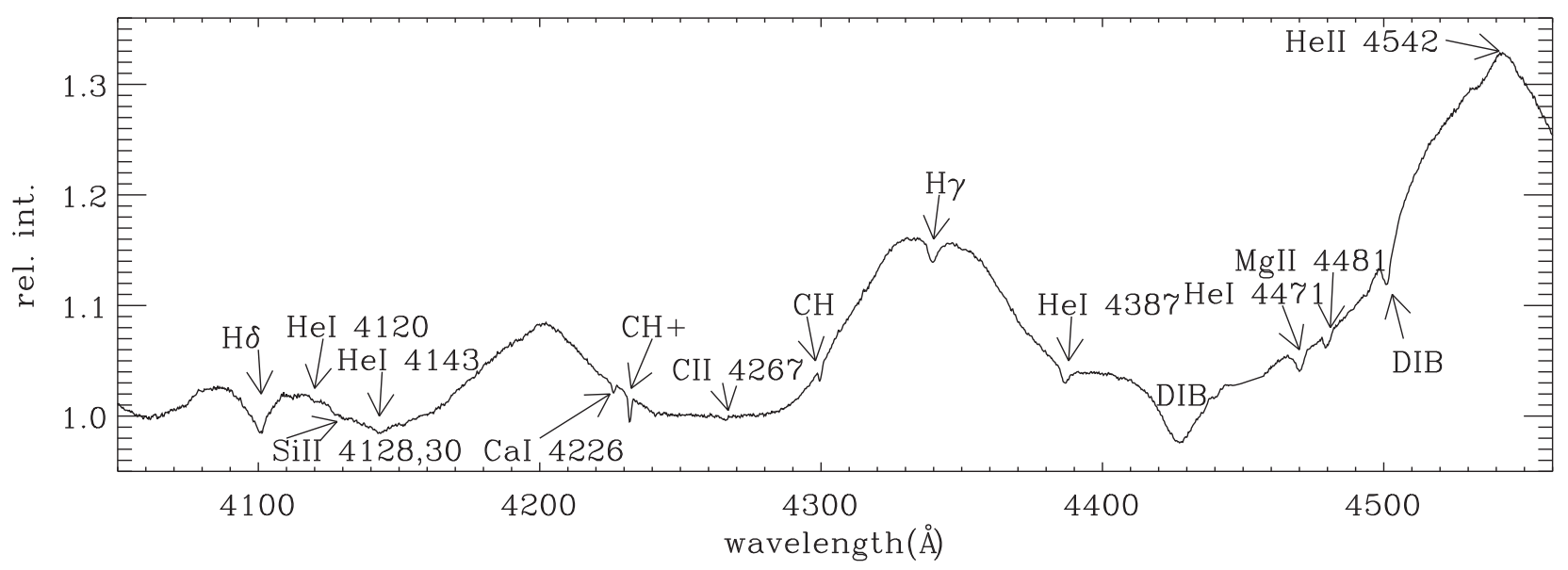

Figure 1. High S/N average GMOS spectrum obtained between 2012 July 11 and August 31. We show here only the wavelength range between $4050 \AA ̊$ and $4560 \AA$, where most of the absorption lines are visible. We label the IS lines as well as the H, He I, Si II, C II, and Mg II lines seen superimposed on the WR emission lines (unlabeled). Note the absence of a narrow absorption line at He II $\lambda 4542$.

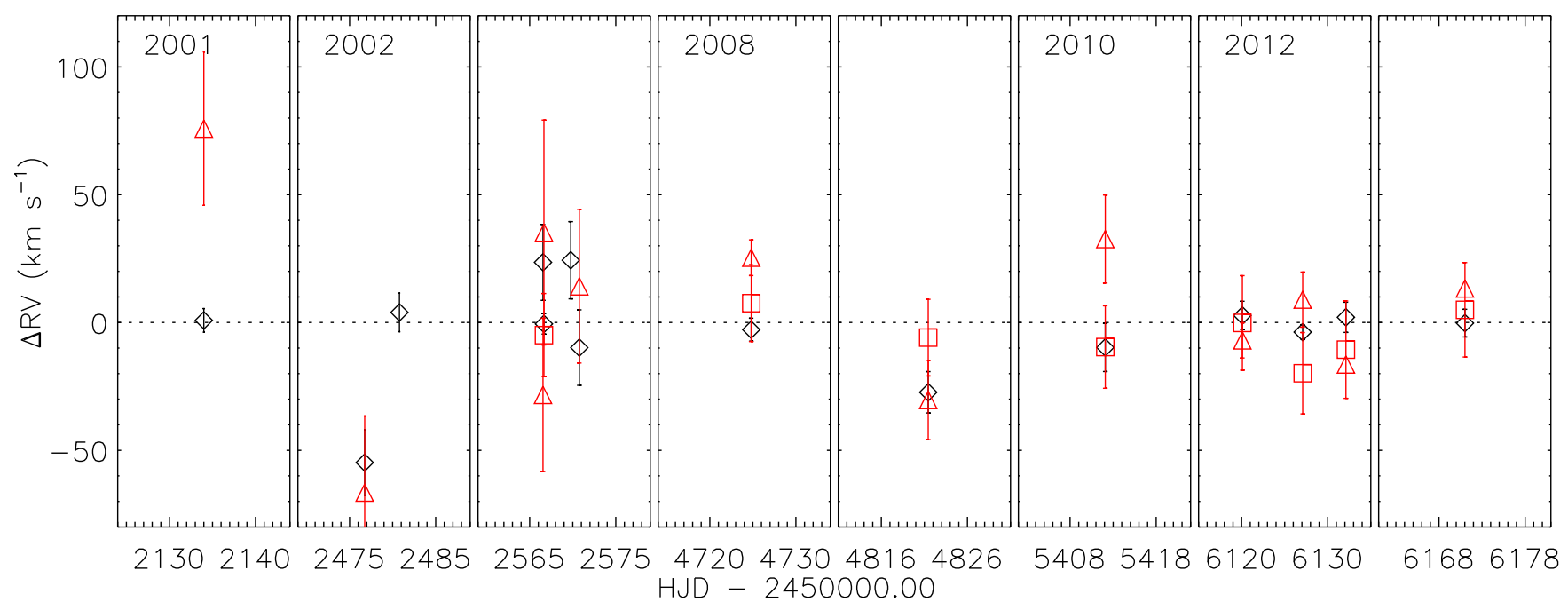

Figure 2. Radial velocity (RV) variations of WR 2 measured using all our spectra observed between 2001 and 2012. The emission line RV shifts (black diamonds) were obtained by cross-correlating the spectra with the GMOS spectrum of 2012 August 31 that served as a reference. The absorption line RVs were measured by fitting their profiles using a Voigt function. We assume that the lines of a given element will all move together, hence, we combine the RVs for all $\mathrm{H}$ (red triangles) and He lines (red squares). The $\pm 1 \sigma$ error bars associated with each measurement are superposed on the data points.

Rate \& Crowther (in preparation), we find the distance for members with Gaia parallaxes (see Fig. 4) and calculate a mean of $2.45 \mathrm{kpc}$. However, this does not account for the increased error bar size of BD + 60169 and other more distant members. Taking this into consideration, we use a distance of $2.4 \mathrm{kpc}$ for the association centre and WR 2 .

We have performed a preliminary atmospheric model of the optical spectrum of WR 2 using CMFGEN (Hillier \& Miller 1998), which solves the transfer equation in the co-moving frame subject to statistical and radiative equilibrium, assuming an expanding, spherically symmetric, homogeneous, and static atmosphere, allowing for line blanketing and clumping. CMFGEN does not solve the momentum equation, so the supersonic velocity is parameterized with a classical $\beta$-type law. For this model, we adopted the standard value for the exponent of $\beta=1$. The model yielded an interstellar reddening of $E(B-V)=0.45 \mathrm{mag}$ for an adopted $R_{V}=3.1$ extinction law (Seaton 1979), corresponding to $E(b-v)=0.34$ mag in the $u b v r$ WR filter system. WR2 has an apparent magnitude of $v=11.33$, so its absolute magnitude is $M_{v}=-2.1$ mag for our adopted distance.

By comparing our mean GMOS spectrum to our WN2 model atmosphere, we estimate an $\mathrm{H} \gamma$ equivalent width of $W_{\lambda}$ $=0.30 \pm 0.05 \AA$ for the B star. From Millward \& Walker (1985), mid-B dwarfs have $\mathrm{H} \gamma$ equivalent widths of $6 \pm 1 \AA$, so the contribution of the B star to the spectrum of WR 2 is approximately 5 percent at the wavelength of $\mathrm{H} \gamma$. Since $M_{v}=-1.9 \pm 0.5 \mathrm{mag}$ for B2-4 V stars (Millward \& Walker 1985), one would expect a 50 per cent contribution if the B star was physically associated with the WN star, or it lay at the same distance. Therefore, assuming the distance to WR 2 is correct, it is much more likely that the B star is a background line-of-sight companion to WR 2. Such a conclusion is also supported by the 2.4 mag difference in the i' band between WR 2 and the B star, giving a distance to the B star of $4 \mathrm{kpc}$, at a distance of $160 \mathrm{pc}$ from the Galactic mid-plane. While this may seem unlikely, we also note that a $\mathrm{B} 2.5 \mathrm{~V}$ star has a mass of only $6 \mathrm{M}_{\odot}$, so it is plausible. In Fig. 5, we present archival ultraviolet 


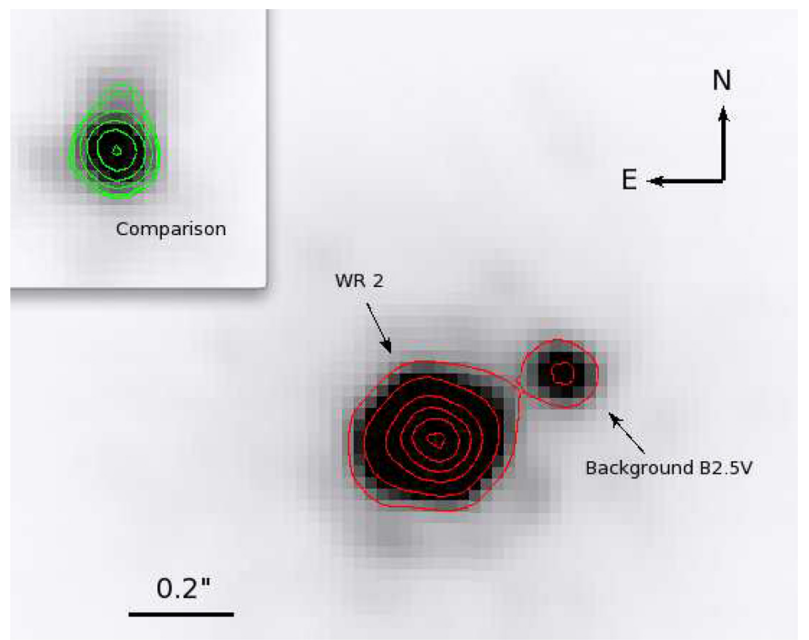

Figure 3. $K$-band diffraction limited image of WR 2 obtained with NIRI + Altair at Gemini-North. The contours mark levels between 10 and $15000 \mathrm{e}^{-} \mathrm{s}^{-1}$ increasing in a square root scale. The star TYC4017-1829-2, situated 13.5 arcsec South of WR 2 is also shown in the left top corner for comparison.

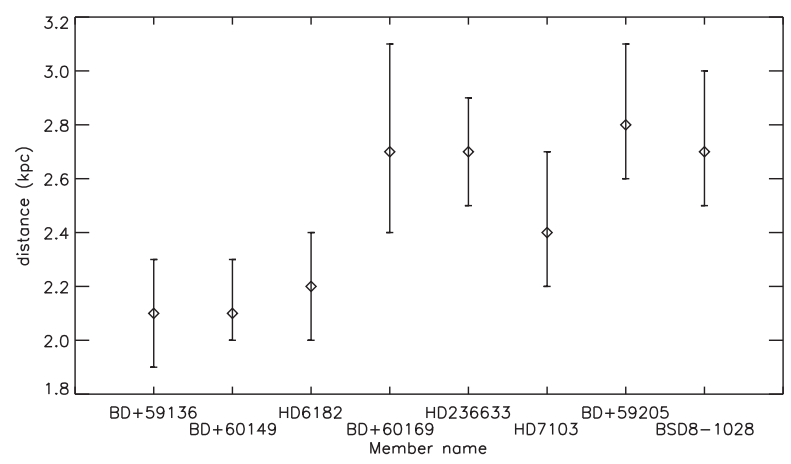

Figure 4. Distance for the Cas OB1 association members using Gaia parallaxes.

(IUE/LORES) and optical (INT/IDS) spectrophotometry of WR 2 together with our reddened atmospheric model for the WN2 star and a Kurucz ATLAS9 model with parameters representative of a mid-B dwarf $\left(T_{\text {eff }}=19,000 \mathrm{~K}, \log g=4.0\right)$ scaled to 5 percent of the WN star. Of course, since the B star is a background star, its interstellar reddening would potentially be significantly higher than that of the WN star.

We conclude that the B-star continuum does not significantly dilute the WR 2 spectrum and cannot be the principal cause of the emission lines' unusual shape.

Interestingly, the spectral line amplitudes obtained by our model correspond roughly to those observed, even without the significant contribution of a companion's continuum. This could be a manifestation of the Baldwin effect (van Gent et al. 2001), i.e. an overall dilution of the WR star's emission lines, as we confirm that WR 2 has a small radius and a high temperature, potentially leading to a quite strong effect. On the other hand, the shape of the line profile remains unusual, as the models give broad Gaussian-like profiles, in stark contrast with the observed round-shaped lines. We therefore maintain that other phenomena need to be explored to explain fully the WR 2 spectrum.

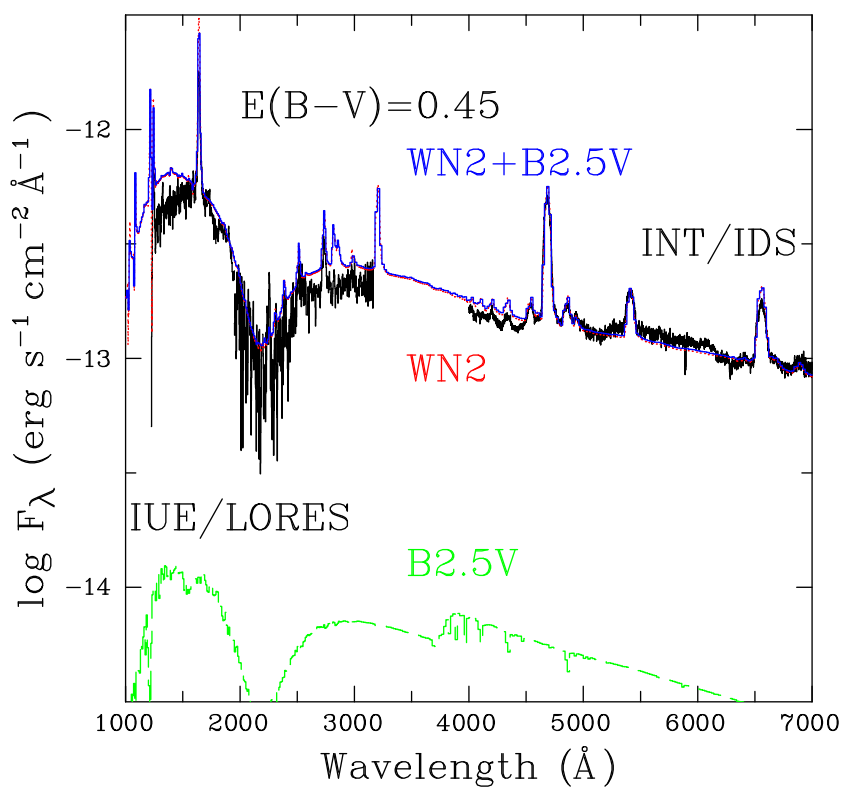

Figure 5. Observed spectral energy distribution of WR 2 (IUE/LORES, INT/IDS) together with models for the WN2 star (red, dotted line), B2.5 V (green, dashed lines) and combined system WN2 + B2.5 V (blue, solid line), reddened with $E(B-V)=0.45$ mag.

\section{SEARCH FOR EVIDENCE OF RAPID ROTATION}

Rapid stellar rotation would cause the geometry of WR 2 and its wind to depart from spherical symmetry. This can be revealed by a component of intrinsic linear polarization, measurable either in broadband polarimetry or spectropolarimetry. Continuum - and some high-ionization line - photons emitted in the inner part of the wind will be scattered by free electrons, and polarized. In a spherically symmetric wind, the individual (vectorial) contributions cancel out. However, if spherical symmetry is broken, as is the case, for example for a rotationally deformed, peanut-shaped or equatorially flattened wind (Maeder 1999) or any local wind density structure, the net continuum polarization will be non-zero. Emission lines of lower ionization, on the other hand, mostly form through recombination further out in the wind, and therefore are less or even not at all polarized. This leads to the reduction in observed polarization across the emission lines with respect to the adjacent continuum, also called the 'line-effect' (see Harries, Hillier \& Howarth 1998). This line effect is thus a very sensitive and highly reliable diagnostic for asymmetric winds in massive stars (e.g. StLouis 2013). Even in the case of a spherically symmetric rotating wind, Harries (2000) has shown that a line effect can be produced because of the rotational velocity field. For parameters appropriate for the O star $\zeta$ Puppis $\left(v_{\text {rot }}\right.$ (equator) $\sim 200 \mathrm{~km} \mathrm{~s}^{-1}$ ), he predicts a line depolarization level of 0.1 per cent.

\subsection{Observations}

\subsubsection{Linear spectropolarimetry}

A spectrum in polarized light of WR 2 in the three Stokes parameters I, Q, and U was obtained on 2010 August 3 with ESPaDOnS at the CFHT. The spectra were reduced by the pipeline LibreESpRIT (Donati et al. 1997). Each observed spectrum reached a $\mathrm{S} / \mathrm{N} \sim 170$ per resolution element $\left(2.6 \mathrm{~km} \mathrm{~s}^{-1}\right)$ around $5000 \AA$. Since 
WR winds have typical turbulent root mean square ( $\mathrm{rms}$ ) velocities of $v_{t} \sim 100 \mathrm{~km} \mathrm{~s}^{-1}$ in the radial direction (Lépine \& Moffat 1999) (much less in the tangential direction), we chose to bin several resolution elements to obtain a higher $\mathrm{S} / \mathrm{N}$. To reach a precision of 0.03 per cent in the Stokes parameters, we binned the spectra to reach a $\mathrm{S} / \mathrm{N} \sim 3000$ per bin, which had typical sizes between 800 and $1500 \mathrm{~km} \mathrm{~s}^{-1}$ in the continuum. Since the peak of the He II $\lambda 4686$ line has an intensity 3.5 times higher than the continuum, the spectra need a binning factor that is $\sqrt{3.5}$ times smaller at line peak than in the continuum to reach identical $\mathrm{S} / \mathrm{N}$ per bin and at the same time produce a reasonable number (15) of bins.

One should note that even though ESPaDOnS allows us to obtain reliable polarization relative to the adjacent continuum, this instrument is not designed to obtain accurate measurements of the polarization of the continuum itself (e.g. Moffat \& Eversberg 2000). On the other hand, ESPaDOnS has already been used successfully to detect line-effects in the spectrum of WR 1 using the relative Q and U Stokes parameters (St-Louis 2013).

\subsubsection{Broadband polarimetry}

We obtained linear polarimetry of WR2 using the 'La Belle et la Bête' polarimeter at the Mont Mégantic Observatory (see Manset \& Bastien 1995) on the nights of 2010 October 18 and 19. We used three broadband filters (with widths of c. $1000 \AA$ at $\lambda_{\mathrm{c}}=7500,5500$, and $4300 \AA$ ), as well as a medium-band filter at $4700 \AA$ with a bandpass of $100 \AA$. The latter region is dominated by the strong He II $\lambda 4686$ emission line. We also observed two polarized (HD 7927 and HD 19820) and two non-polarized ( $\beta$ Cas and HD 21447) standards in all filters, except the medium-band one where the flux was too low to make this feasible. The former were used to calibrate the polarization angles, while the latter yielded insignificant instrumental polarization at the 0.02 percent level. The polarization efficiency ( 80 per cent) was measured in all four filters using a prism which polarizes the light at 100 percent at all optical wavelengths.

The observations of HD 7927 indicate that the polarization angle does not change significantly with wavelength at the $1^{\circ}$ level, which leads us to believe that the angle is also the same in the medium-band filter. On the other hand, unlike HD 7927, the polarization angle for HD 19820 deviated from its reported standard value by $50^{\circ}$ in all the observed bands, the origin of which remains unexplained.

\subsection{Search for a line effect}

Fig. 6 shows the relative $Q$ and U Stokes parameters obtained for the He II $\lambda 4686$ line, the strongest emission line in the optical band of WR 2. The mean intensity profile is shown in the bottom panel of the figure. There is no clear signature in the emission lines of a line-effect due to the dilution of polarized continuum light by unpolarized line flux. Harries et al. (1998) carried out Monte Carlo simulations to compute polarization spectra of a wind with a latitude dependent density distribution. For the HeII $\lambda 5412$ line, they found that an equator-to-pole density contrast of 1.5 produces depolarization level of $\sim 0.1$ per cent. When significant continuum polarization levels were detected for their sample of stars through the line effect, they report values of 0.6 per cent, corresponding to an equator-to-pole density ratio of $2-3$. Our non-detection, with a $1 \sigma$ error bar of 0.05 per cent, corresponds to an equator-to-pole density contrast in their model of 1.2, leading us to conclude that the wind is highly symmetric with no direct sign of a wind asymmetry that

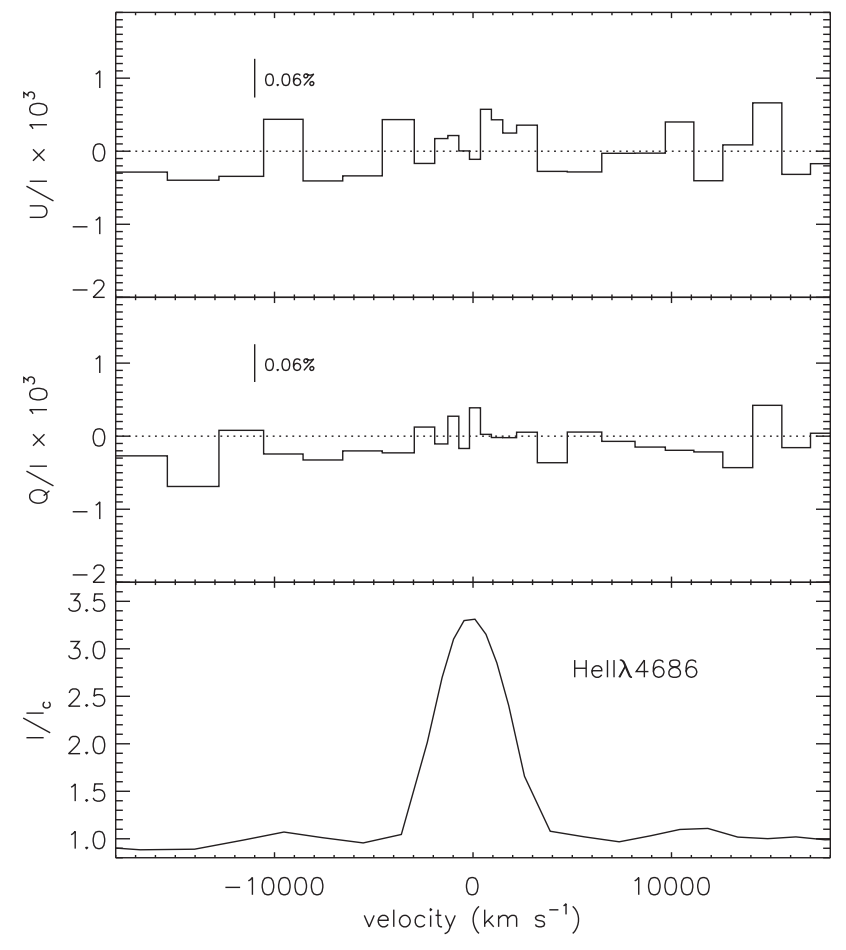

Figure 6. Relative Stokes parameters $\mathrm{I} / \mathrm{I}_{\mathrm{c}}$ (bottom), Q/I (middle) and U/I (top) for the He II $\lambda 4686$ line of the hot WN star WR 2. The $1 \sigma$ error bars are indicated in the top left corner of the top two panels.

Table 2. Linear broadband and medium-band polarimetry of WR 2 .

\begin{tabular}{lccccc}
\hline$\lambda$ & $(\AA)$ & 7500 & 5500 & 4300 & $4700 / 100$ \\
\hline $\mathrm{P}$ & $(\%)$ & $3.07(6)$ & $3.45(8)$ & $3.83(10)$ & $3.24(25)$ \\
$\theta$ & $(\mathrm{deg})$ & $40(1)$ & $35(1)$ & $37(1)$ & $47(2)$ \\
\hline
\end{tabular}

could indicate rapid stellar rotation. The absence of a line effect in our case also indicates the absence of a significant rotational velocity field (Harries 2000). This contradicts the assertion that the wind of WR 2 might have a rotation velocity of $1900 \mathrm{~km} \mathrm{~s}^{-1}$, which is near the break-up velocity and would most likely lead to a very strong wind asymmetry.

\subsection{Continuum linear polarization and Serkowski fit}

There is a wavelength dependence of linear polarization seen in essentially all stars that is caused by the ISM, and which follows a Serkowski law (Serkowski et al. 1975). We are therefore searching for an intrinsic component of polarization due to electron scattering, which is independent of wavelength in the non-relativistic case. For WR 2, the final reduced values of the polarization are summarized in Table 2. We fit simultaneously the 3 broad-band values of $\mathrm{Q}=$ $\mathrm{P} \cos 2 \theta$ and $\mathrm{U}=\mathrm{P} \sin 2 \theta$ to equations (21) and (22) of Moffat \& Piirola (1993) for ISM and intrinsic polarization, with six free parameters. Only six available data points ( $Q$ and $U$ for three bands) are not enough to properly fit all the parameters in a meaningful way. Still, after an initial trial, we found that the angle of the ISM polarization $\left(\theta_{\text {ISM }}\right)$ does not depend strongly on wavelength, and that in the equation $\theta_{\text {ISM }}=\theta_{\text {ISM }}(0)+k / \lambda$, we could assume $k=0$ and reduce the number of parameters to fit down to 5 . This first simplification helped, but the fit still remains poorly constrained, as many solutions could satisfy a $\chi^{2}$-minimization algorithm. 


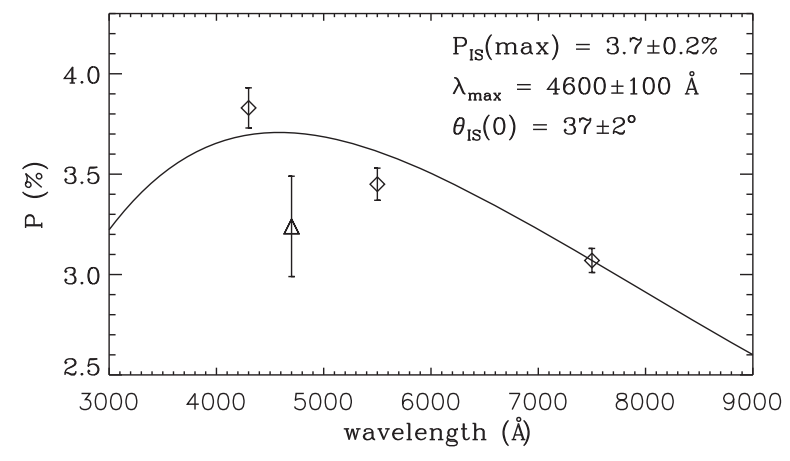

Figure 7. Broadband polarization observations of WR 2 (diamonds) superposed on a fitted standard Serkowski law (Serkowski, Mathewson \& Ford 1975). The narrowband filter observation including the He II $\lambda 4686$ line is also included (triangle).

In order to check if the intrinsic polarization of WR 2 is compatible with a value of zero, as suggested by the linearly polarized spectra discussed in the previous sub-section, we determine what result is obtained when we carried out the fit with an imposed null intrinsic stellar polarization (independent of wavelength). We therefore fitted only three parameters: the maximum ISM polarization, $P_{\mathrm{ISM}}(\max )$, the wavelength at $P_{\mathrm{ISM}}(\max ), \lambda_{\max }$ and $\theta_{\mathrm{ISM}}$. The value we obtained for $\lambda_{\max }$ is $4600 \pm 100 \AA$, which is blueward of the average Galactic value that is close to $5500 \AA$. This could simply imply smaller grains on the average towards WR 2. The measured values of $P$ are plotted in Fig. 7 as a function of wavelength. The fitted Serkowski law (Serkowski et al. 1975), giving the theoretical curve of the ISM polarization, is overplotted. It can be seen that the measurements can be reproduced solely with ISM polarization and that there is no need for a non-zero intrinsic polarization of WR 2. Considering that the reddening for WR 2 is $E(b-v)=0.4 \mathrm{mag}$, the maximum interstellar polarization expected is $P_{\mathrm{ISM}}(\max )=3.6$ per cent, according to Draine (2003)'s ISM law. This agrees very well with our findings of $P_{\mathrm{ISM}}(\max ) \sim 3.5$ percent. The narrowband filter containing the strong He II $\lambda 4686$ emission line deviates only by $2 \sigma$ from the theoretical curve at this level. This is compatible with the spectropolarimetric result above, which indicates that there is no significant line depolarization. Nevertheless, higher precision linear pol measurements with better wavelength coverage would be important for more firmly constraining this result.

\section{SEARCH FOR EVIDENCE OF A MAGNETIC FIELD}

Shenar et al. (2014) predict that WR 2 hosts a very strong, organized magnetic field ( $40 \mathrm{kG}$ at $R=R_{*}$ and $14 \mathrm{kG}$ at $R=1.7 R_{*}$ ) to enforce the co-rotation required to explain the round-top line profiles in their model. Circular polarimetry can be used to detect magnetic fields via the Zeeman effect in polarization. Using ESPaDOnS at CFHT, we obtained a spectrum in polarized light in the Stokes V parameter on 2014 January 9, as part of the BinaMIcS ${ }^{2}$ (Binarity and Magnetic Interactions in various classes of Stars) survey.

\footnotetext{
${ }^{2}$ BinaMIcS is an international project led by France (PI E. Alecian), which aims to exploit binarity to yield new constraints on the physical processes at work in hot and cool magnetic binary and multiple systems (Alecian et al. 2014).
}

\subsection{Stokes V (circular) spectropolarimetry}

Fig. 8 shows in the top panels the V/I signal (plotted with a solid black line) we obtained for four emission lines, i.e. He II $\lambda \lambda 4542$, 4686,5411 , and 6562 . The bottom panels show the lines' mean profile. The original $\mathrm{S} / \mathrm{N}$ was 100 per resolution element $\left(1.8 \mathrm{~km} \mathrm{~s}^{-1}\right)$ around $5000 \AA$, but we binned the spectra so that they reached a S/N of 2000,3500,3500, and 4500 per bin for the four lines, respectively (note that the ESPaDOnS throughput is better towards the red). The observations do not show any clear or coherent signal above $1 \sigma$ (the uncertainties are plotted as a vertical line in each case). To estimate if our lack of detection of a Stokes V/I signal in those lines is compatible with the values predicted by Shenar et al. (2014), we compare our observations to the split-monopole model for a global magnetic field in a WR wind of Gayley \& Ignace (2010). For a magnetic pole-on inclination (other inclinations are not considered, but would give qualitatively similar or lower amplitude results) and for optically thick (Sobolev optical depth in the line is much greater than 1) recombination lines ( $p=6)$, Gayley \& Ignace (2010) find (their equation 30 and 32 and in last paragraph of their Section 2.2 as well as their Fig. 3):

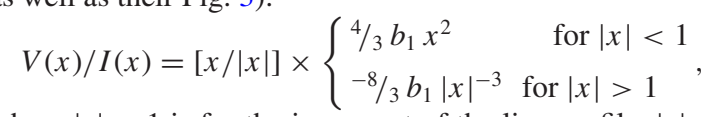

where $|x|<1$ is for the inner part of the line profile, $|x|>1$ is for the wings and $x=\left[\lambda-\lambda_{0}\right] / \lambda_{0} . x$ is \pm 1 for $\lambda-\lambda_{0} \sim \pm$ HWHM of the line, and :

$$
b_{1}=7.7 \times 10^{-4} g_{\text {eff }}\left(\lambda_{0} /{ }_{5500 \AA}\right) \frac{B(r) / 100 G}{v(r) / 100 \mathrm{kms}^{-1}} \text { for } r=1,
$$

is a factor depending on the magnetic field strength and corresponding to where the line emission peaks.

In Section 2.5, we described briefly our CMFGEN (Hillier \& Miller 1998) model for WR 2. From the resulting model we obtained the emitting region of the different lines. In Fig. 9, we present our results using $\beta=1$ and $v_{\infty}=3200 \mathrm{~km} \mathrm{~s}^{-1}$ (obtained from our CMFGEN model).

We find from Fig. $9, v(r)=2500 \mathrm{~km} \mathrm{~s}^{-1}$ for the peak emission of the He II $\lambda 4686$ line, and thus we adopt that the line emission region is centred at a distance of $R=4.6 R_{*}\left(=3.9 \mathrm{R}_{\odot}\right)$ from the centre of the star. Taking $B(r)=1900 G$ at $4.6 R_{*}$ (scaling as $r^{-2}$ from the $40 \mathrm{kG}$ value at the stellar surface - Shenar et al. 2014) and $g_{\text {eff }} \sim 1$, we obtain $b_{1}=0.00050(0.050$ per cent $)$. Using this value, we overplot the modelled $V(x) / I(x)$ versus $\lambda$ (with a green dotted line) in Fig. 8 on the data. However, for a fair comparison, we bin the model to the same velocity sampling as the observations (red dashed lines). From this plot, we see that the predicted magnetic signal, that sometimes barely reaches $2 \sigma$, is comparable to the observational uncertainties. The results are therefore inconclusive in the context of the Shenar et al. (2014) study.

On the other hand, the magnetic field inferred for WR 2 by Shenar et al. (2014) assumes a split monopole solar wind-type model (Weber \& Davis 1967) for the field from the stellar surface to the Alfven radius. As discussed by ud-Doula, Owocki \& Townsend (2009, Sections 2 and 3), the split monopole model is an idealized case, allowing a quasi-analytic formulation that is not possible with a more realistic dipole field.

The assumption of a split monopole field and its corresponding $r^{-2}$ decay beginning at the stellar surface is conceptually at odds with the picture of a rigidly rotating magnetosphere in which the wind is confined by the field out to $r=R_{\mathrm{A}}=6 R_{*}$. In fact, the split monopole geometry of Weber \& Davis (1967) is a simplification which explicitly assumes that the wind dominates the magnetic field at the stellar surface. However, in the picture adopted by Shenar et al. 

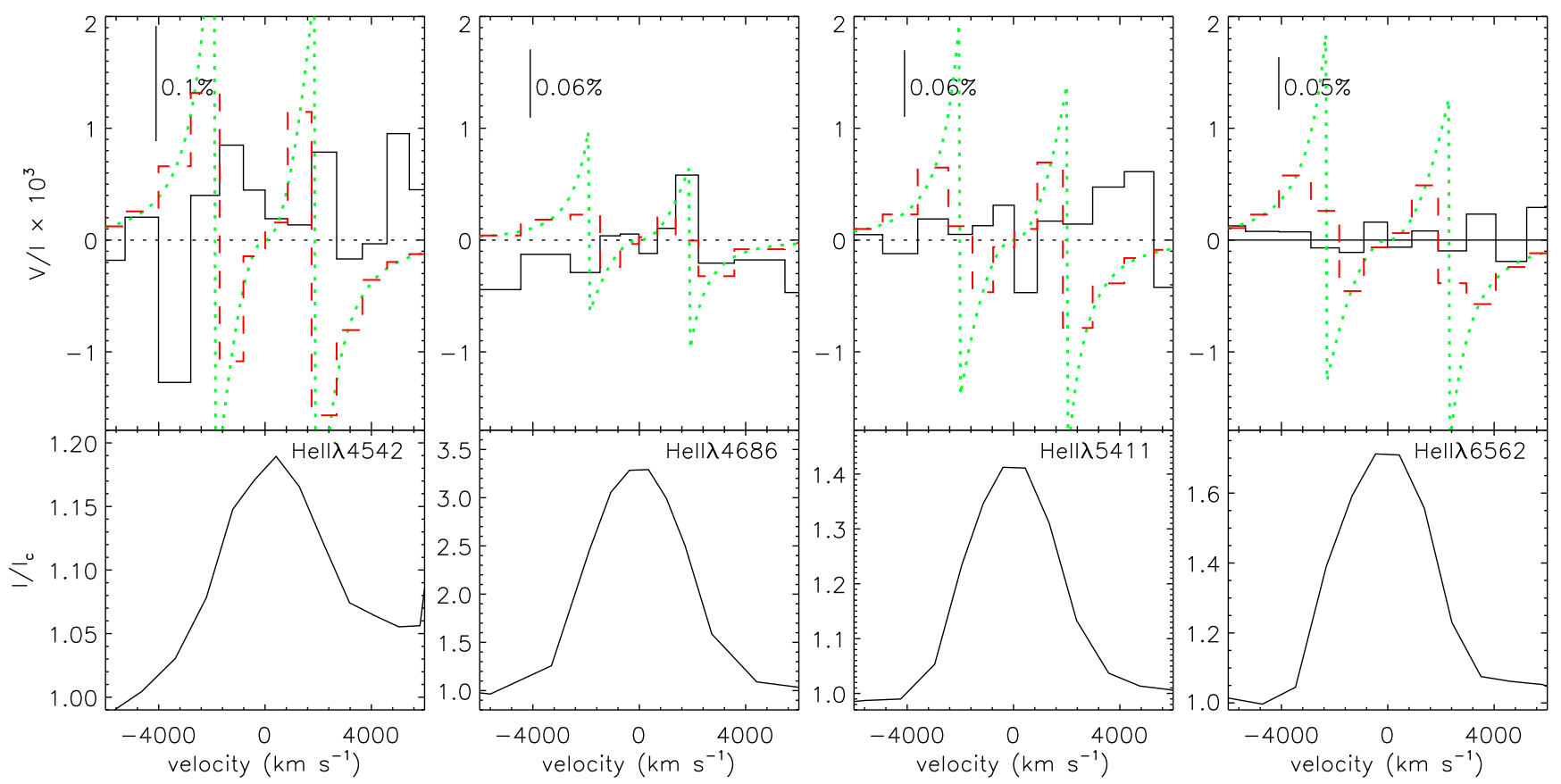

Figure 8. Top panels: Observations (black solid line) compared with the split-monopole model (green dotted line) of $V(x) / I(x)$ versus $\lambda$ of Gayley \& Ignace (2010). For a better comparison, the model is also binned into the same velocity sampling as the observations (red dashed line). The $\pm 1 \sigma$ error bar is indicated in the top left corner. Bottom panels: Mean line profiles.

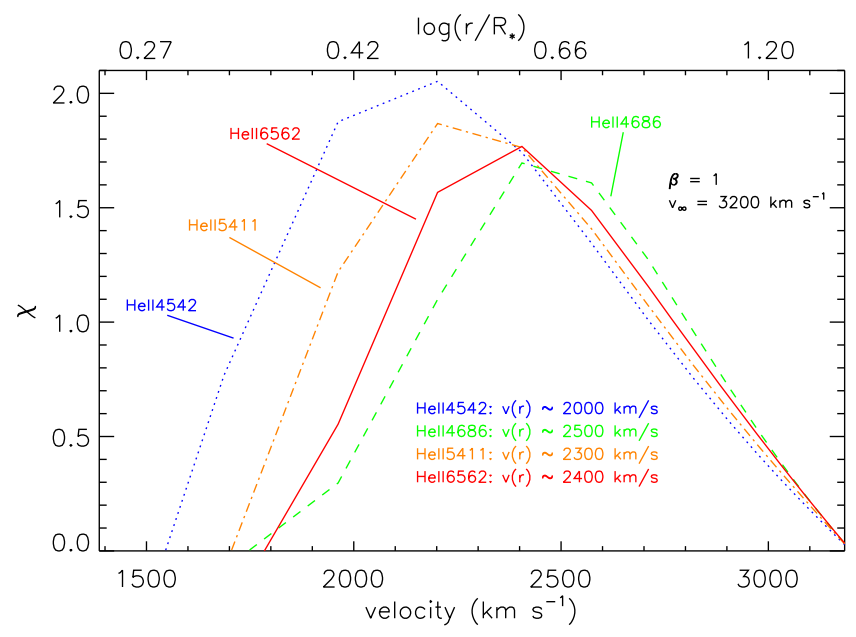

Figure 9. Line formation region emission $(\chi)$ calculated with the CMFGEN code for the He II $\lambda 4542,4686,5411$, and 6562 line profiles. The value $\chi$ is plotted as a function of the radius, and also as a function of velocity defined as $v=v_{\infty} *\left(1-\frac{R_{*}}{r}\right)^{\beta}$, where $R_{*}$ is the WR 2 stellar radius. The value $v(r)$ (given in the plot) is the velocity at which maximum emissivity occurs.

(2014), the field must be essentially undistorted by the wind out to the Alfven radius.

Another discordance with previous works in the derivation of Shenar et al. (2014) is that the wind in a split monopole model corotates up to the Alfven radius. As discussed by ud-Doula et al. (2009), most of the angular momentum loss in such a model is actually from the Poynting stresses in the magnetic field, not in the azimuthal velocity of the wind. Thus, the wind is actually rotating more slowly than the rigid body value at $R_{\mathrm{A}}$. Ultimately, to really force the wind to co-rotate out to $6 R_{*}$ would require a magnetic field at the surface of WR 2 that is substantially stronger than that inferred by Shenar et al. (2014).

\subsection{Revised magnetic field predictions}

In this section, we attempt to revise the prediction of WR2's magnetic field using more up-to-date assumptions. To calculate the required field, we begin by assuming the simplest possible structure of the unperturbed stellar magnetic field: a dipole aligned with the rotation axis. We adopt the formalism of ud-Doula \& Owocki (2002), more specifically, we used equation (4) of ud-Doula et al. (2009) to compute the wind confinement parameter $\eta(r)$ :

$\eta(r)=\eta_{*} \frac{r^{2-2 q}}{(1-1 / r)^{\beta}}$,

where $r$ is in units of $R_{*}, q$ is the power-law exponent for the radial decline of the assumed stellar field, e.g. $q=3$ for a pure dipole, $\beta$ is the velocity-law index of the wind, and $\eta_{*}$ is the wind confinement at the stellar surface. Setting $\eta\left(R_{\mathrm{A}}\right)=1$ and assuming a dipole stellar field, we find that the Alfven radius can be evaluated as the root of the following expression:

$R_{\mathrm{A}}^{4}\left(1-1 / R_{\mathrm{A}}\right)^{\beta}=\eta_{*}$.

[N.B. equation (2) is just a generalization of equation (8) of udDoula, Owocki \& Townsend 2008, to explicitly include the exponent $\beta$.] We evaluate equation (2) for $r=R_{A}=6 R_{*}$, which yields $\eta_{*}=1080$ for $\beta=1$. Adopting $R=7 R_{*}$ for the radius of formation of the bulk of He II $\lambda 4686$ ( $1 R_{*}$ outside of the Alfven radius), we can infer the field required by the rigid rotation model that should be reflected in the circular polarization of this line.

We assume that the magnetic field decays as $r^{-3}$. While this is not strictly true outside the Alfven radius (where the field will be 
ultimately stretched into an approximate split monopole decaying as $r^{-2}$ ), the proximity of the line formation region and the Alfven radius makes this a reasonable approximation. Moreover, it will provide a conservative upper limit on the field required by the model.

According to equation (6) of ud-Doula et al. (2008), and considering the radius $R_{*}=0.85 \mathrm{R} \odot$, mass-loss rate $\dot{M}=10^{-5.3} \mathrm{M}_{\odot} \mathrm{yr}^{-1}$ and wind terminal velocity $v_{\infty}=1800 \mathrm{~km} \mathrm{~s}^{-1}$ (Shenar et al. 2014) of WR 2, $\eta_{*}=1080$ corresponds to a surface dipole strength of $B_{\mathrm{eq}}=\sqrt{\eta_{*} \dot{\mathrm{M}} v_{\infty} / R_{*}^{2}}=130 \mathrm{kG}$. For comparison, Shenar et al. (2014) estimated a surface field strength of just $40 \mathrm{kG}$ (with $\beta=1$ ).

Extrapolating these surface fields to $r=7 R_{*}$ according to the scheme described above, we estimate $B\left(7 R_{*}\right)=380 \mathrm{G}$ for $\eta_{*}=1080$. Fig. 8 illustrates that this field is well below the noise level of our circular polarization data.

It is thus clear that even with the much stronger surface fields predicted by our more realistic model of the wind confinement of WR 2, the more rapid decay of the field strength with distance results in fields in the line formation region that are of comparable strength to that predicted by Shenar et al. (2014). Our observations are unfortunately of insufficient quality to confirm or falsify this revised prediction.

However, our revised model still has important implications for the plausibility of the conclusions derived for WR 2 and the other stars studied by Shenar et al. (2014). First, in a manner similar to WR 2, the surface field strengths required for the other stars must also be substantially stronger for this mechanism to work. For example, to enforce co-rotation of the wind of BAT99-7 (one of the most extreme cases of broad-rounded emission lines in the LMC) out to $16 R_{*}$ would require a surface field of about $12 \mathrm{MG}$ (megagauss!) for $\beta=1$. Such a field corresponds to a surface magnetic flux of $2.5 \times 10^{8} \mathrm{GR}_{\odot}^{2}$, which is vastly larger than those typically inferred in strongly magnetized upper main sequence stars, white dwarfs and neutrons stars $\left(\lesssim 1 \times 10^{6} \mathrm{GR}_{\odot}^{2}\right)$.

Secondly, angular momentum loss due to the strongly magnetized winds of these stars is difficult to reconcile with their inferred rotation speed. In the case of WR 2, the spindown e-folding timescale $\left(\tau_{\text {spin }}\right)$ according to equation (25) of ud-Doula et al. (2008), assuming a moment of inertia factor $k=0.1$ ) is just $15000 \mathrm{yr}$. For BAT99-7, it is only $150 \mathrm{yr}$ !

Finally, the rapid rotation and strong magnetic confinement implied by these models would place these stars in the 'Centrifugal Magnetosphere' regime of the Rotation-Confinement diagram of Petit et al. (2013). If the axes of their magnetic fields are oblique to their rotation axes (as is almost always the case in magnetic massive stars, e.g. Grunhut et al. 2012), one would expect significant modulation of the magnetospheric emission signatures according to their rotation periods (approximately $0.1 \mathrm{~d}$ for WR $2,0.4 \mathrm{~d}$ for BAT99-7) as observed for other magnetic O stars (e.g. Howarth et al. 2007). While the velocity regime of the formation region of the He II $\lambda 4686$ emission line and the other lines investigated above lies outside of the magnetospheric region, their zone of formation illustrated in Fig. 9 extends significantly into the magnetosphere, and hence these lines should be subject to important rotational modulation. No such modulation is visible on the time-scale of minutes to years in the spectra used for the analysis in Section 2.3.

\section{SUMMARY AND CONCLUSIONS}

Our observations and analysis do not support any of the presented scenarios to explain the 'bowler-hat'-shaped broad emission lines as observed in WR 2, or any implication in four other WR stars in the LMC. Our conclusions are summarized below.

\subsection{Line dilution from a companion}

We have demonstrated that the WR spectrum of WR 2 is slightly diluted by that of a B-star, but far from enough to explain its spectral lines profile shape.

The search for RV variations did not show significant Doppler motion of the two components for over $10 \mathrm{yr}$. Both stars, separated by 0.25 arcsec, were resolved in our diffraction-limited images, and the B-star is 2.5 and 3 mag fainter than WR 2 in the optical and NIR, respectively. Considering its brightness, we conclude that it is not bound to WR 2, but rather lies fortuitously along its line of sight. Finally, we have found no sign of another star being either bound or close to the line of sight of WR 2 .

One should note that the absence of intrinsic continuum linear polarization is unlike (close) binary WR stars which show phasedependent linear polarization variations and/or variable line depolarization (Harries et al. 1998). Also no signs of wind collision have ever been found for this star. This hence reinforces our conclusion that there is no evidence of the presence of a massive companion bound to WR 2 .

\subsection{Rapid rotation}

We have also demonstrated that the wind of WR 2 is almost certainly spherically symmetric, contradicting the hypothesis that the star is experiencing rapid rotation.

Our ESPaDOnS linear polarization data reveal no line depolarization in four of the strongest lines in the spectrum. This nondetection of line depolarization is compatible with our broadband linear polarization observations: the observed continuum linear polarization can be accounted for solely by the ISM, so the star is unlikely to be intrinsically polarized. This result implies that WR 2 is not affected by the von Zeipel effect (von Zeipel 1924), meaning that its surface is not rapidly rotating. We cannot infer that the core is also not in rapid rotation, since it can be decoupled from the surface. Therefore, we cannot conclude with certainty that WR 2 will never become a LGRB, but since we do not find signs of fast rotation at the surface, there is no reason to consider WR 2 as a LGRB progenitor. Of course, we cannot exclude the unlikely case that we are viewing the rotation axis pole-on and that therefore, the high degree of symmetry we are finding is only apparent. Indeed, assuming that the linear polarization scales with $\sin ^{2} i$ (Brown \& McLean 1977), where $i$ is the viewing angle, the expected line depolarization could be as low as 0.1 per cent ( $2 \sigma$ for our observations with ESPaDOnS) for $i=30^{\circ}$. However, as discussed by Kurosawa, Hillier \& SchulteLadbeck (1999), assuming a sample of randomly oriented axes, the probability of observing a star with a viewing angle less than a given $i_{\mathrm{o}}$ is proportional to the integral over solid angle from $i=0$ to $i=i_{\mathrm{o}}$, where $i$ is the polar angle. Hence, the probability $P\left(i<i_{o}\right)$ for observing a star less than a given $i_{\mathrm{o}}$ is $P\left(i<i_{o}\right)=1-\cos i_{\mathrm{o}}$, and for $i_{\mathrm{o}}=30^{\circ}, P\left(i<i_{\mathrm{o}}\right)=13$ per cent. Moreover, we did explore the possibility to increase the $\mathrm{S} / \mathrm{N}$ up to 3000 per bin for both $Q$ and $U$ Stokes parameters, which gave fewer than 10 bins in the He II $\lambda 4686$ line that became, therefore, relatively poorly resolved. Still, even with an improved signal per bin, none of the bins displayed a $Q$ or a $U$ value that exceeded the $2 \sigma$ error bars, which are in that case 0.06 per cent. Such a shallow line depolarization is obtained for an inclination of $i \sim 7^{\circ}$, which corresponds to $P\left(i<7^{\circ}\right)=0.6$ per cent. Finally, the possibility that WR 2's apparent symmetry is due to the star's inclination can 
easily be verified definitively by observing the linear polarization of the other 'round-lined' WR stars first identified in the LMC by Rühling (2008) (an effort that is beyond the scope of this study).

As an additional note, if WR 2 was a fast rotator, it would make it a prime candidate for strange mode pulsations (SMPs). Indeed, the most violent SMPs are expected in classical WR stars, and are more likely to occur in fast rotating stars (Glatzel et al. 1999). Recent attempts in detecting cyclic photometric variability with periods ranging from minutes to hours, signs of the presence of SMPs, led to a non-detection (Chené et al. 2018).

\subsection{Strong magnetic field}

Shenar et al. (2014) claim that WR 2's round-top emission lines require relatively fast rotation along with a strong magnetic field, $B \sim 14 \mathrm{kG}$ at $\tau=2 / 3$. While our observations are not compatible with fast rotation, we still need to verify the strength of WR 2's magnetic field.

Our new Stokes V spectropolarimetry unfortunately cannot be used to test that hypothesis, as the noise is comparable to the signal we are looking for. Future observations with better S/N are needed. On the other hand, we note that many assumptions used by Shenar et al. (2014) are inconsistent with the more recent efforts in understanding magnetic wind confinement of massive stars. Adopting a more realistic dipole field geometry leads to significantly larger (but still undetectable with the data in hand) predicted surface field strengths that are difficult to reconcile with the inferred and observed properties of WR 2 (and the other WR stars studied by those same authors).

On a side note, if WR 2's predicted field was local, rather than global, it would not be easily detected using spectropolarimetry. But since it would generate strong stellar spots, we could expect line profile variability caused by so-called co-rotating interacting regions (CIRs). Perturbations at the stellar surface, such as (magnetic) bright spots, propagate into the wind and, as they are carried around by rotation, create spiral-like density structures (Cranmer \& Owocki 1996). However, only 20 percent of WR stars show detectable line profile variability interpretable as CIRs (St-Louis et al. 2009; Chené \& St-Louis 2011b) and WR 2 is not one of them. Our spectroscopic analysis in Section 2.3 corroborate the absence of CIRs. Moreover, a local field is not consistent with the large-scale wind confinement needed by the Shenar et al. (2014) model.

\subsection{Future work}

We plan in the near future to reassess the physical and wind parameters of WR 2, and look if an alternative solution with only modest rotation and magnetic field, as suggested by our data, can reasonably reproduce the line profiles, in contrast to the model proposed by Shenar et al. (2014).

\section{ACKNOWLEDGEMENTS}

ANC gratefully acknowledges support Gemini Observatory, which is operated by the Association of Universities for Research in Astronomy, Inc., under a cooperative agreement with the NSF on behalf of the Gemini partnership: the National Science Foundation (United States), the National Research Council (Canada), CONICYT (Chile), the Australian Research Council (Australia), Ministério da Ciência, Tecnologia e Inovação (Brazil) and Ministerio de Ciencia, Tecnología e Innovación Productiva (Argentina). AFJM, NSL, and GAW acknowledge financial support from the
Natural Sciences and Engineering Research Council (NSERC) of Canada. NDR gratefully acknowledges his Centre de Recherche en Astrophysique du Québec (CRAQ) Fellowship. ANC and GAW finally gratefully thank A. de la Chevrotière and S. P. Owocki for discussions and input that led to the significant improvement of this manuscript. NDR acknowledges postdoctoral support by the University of Toledo and by the Helen Luedtke Brooks Endowed Professorship. The Robo-AO system was developed by collaborating partner institutions, the California Institute of Technology and the Inter-University Centre for Astronomy and Astrophysics, and with the support of the National Science Foundation under Grant Nos. AST-0906060, AST-0960343, and AST-1207891, the Mt. Cuba Astronomical Foundation and by a gift from Samuel Oschin. Ongoing science operation support of Robo-AO is provided by the California Institute of Technology and the University of Hawai'i. C.B. acknowledges support from the Alfred P. Sloan Foundation. CZ is supported by a Dunlap Fellowship at the Dunlap Institute for Astronomy \& Astrophysics, funded through an endowment established by the Dunlap family and the University of Toronto. Based on observations obtained at the Gemini Observatory, processed using the Gemini IRAF package. The program ID are GN-2012B-Q-115 and GN-2014B-Q-109. Based on observations obtained at the Canada-France-Hawaii Telescope (CFHT), which is operated by the National Research Council of Canada, the Institut National des Sciences de l'Univers of the Centre National de la Recherche Scientifique of Francethe University of Hawaii. Also based on obsrvations obtained at the Gemini Observatory, which is operated by the Association of Universities for Research in Astronomy, Inc., under a cooperative agreement with the NSF on behalf of the Gemini partnership: the National Science Foundation (United States), the National Research Council (Canada), CONICYT (Chile), the Australian Research Council (Australia), Ministèrio da Ciència, Tecnologia e Inovação (Brazil), and Ministèrio de Ciència, Tecnología e Innovación Productiva (Argentina).

\section{REFERENCES}

Alecian E. et al., 2015, Proc. IAU Symp. 307, New Windows on Massive Stars: Asteroseismology, Interferometry, and Spectropolarimetry. Geneva, Switzerland, p. 330

Baranec et al., 2014, ApJ, 790L, 8

Brown J. C., McLean I. S., 1977, A\&A, 57, 141

Chené A.-N., Moffat A. F. J., Crowther P. A., 2008, Clumping in hot-star winds, Potsdam, Germany, p.163

Chené A.-N., St-Louis N., A. F. J. Moffat, O. Schnurr, É. Artigau, 2018, Res. Notes Am. Astron. Soc., 2c, 168

Cranmer S. R., Owocki S. P., 1996, ApJ, 462, 469

Crowther P. A., 1993, PhD Thesis, Univ. London

Crowther P. A., 2007, ARA\&A, 45, 177

Donati J.-F., Semel M., Carter B. D., Rees D. E., Collier Cameron A., 1997, MNRAS, 291, 658

Draine, 2003, ARA\&A, 41, 241

Ferrario L., Wickramasinghe D., 2006, MNRAS, 367, 1323

Gaensler B. M. et al., 2005, ApJ, 620, L95

Gayley K. G., Ignace R., 2010, ApJ, 708, 615

Glatzel W., Kiriakidis M., Chernigovskij S., Fricke K. J., 1999, MNRAS, 303,116

Grunhut J. H. et al., 2103, MNRAS, 426, 2208

Hamann W.-R., Gräfener G., Liermann A., 2006, A\&A, 457, 1015

Harries T. J., 2000, MNRAS, 315, 722

Harries T. J., Hillier D. J., Howarth I. D., 1998, MNRAS, 296, 1072

Hillier D. J., Miller D. L., 1998, ApJ, 496, 407

Howarth I. D. et al., 2007, MNRAS, 381, 433

Kelly P. L., Kirshner R. P., Pahre M., 2008, ApJ, 687, 1201 
Kurosawa R., Hillier D. J., Schulte-Ladbeck R. E., 1999, AJ, 118, 539

Law N. M. et al., 2014, ApJ, 791, 35L

Lépine S., Moffat A. F. J., 1999, ApJ, 514, 909

MacFadyen A. I., Woosley S. E., 1999, ApJ, 524, 262

MacFadyen A. I., Woosley S. E., Heger A., 2001, ApJ, 550, 410

Maeder A., 1999, A\&A, 347, 185

Manset N., Bastien P., 1995, PASP, 107, 483

Mel'nik A. M., Dambis A. K., 2017, MNRAS, 472, 3887

Millward C. G., Walker G. A. H., 1985, ApJS, 57, 63

Moffat A. F. J., Piirola V., 1993, ApJ, 413, 724

Moffat A. F. J., Eversberg T., 2000, A\&A, 358, 572

Owocki S. P., ud-Doula A., 2004, ApJ, 600, 1004

Petit V. et al., 2013, MNRAS, 429, 398

Rühling U., 2008, Diploma thesis. Univ. Potsdam

Seaton M. J., 1979, MNRAS, 179, 73P

Serkowski K., Mathewson D. S., Ford V. L., 1975, ApJ, 196, 261

Shenar T., Hamann W.-R., Todt H., 2014, A\&A, 562A, 118

Smith L. F., Shara M. M., Moffat A. F. J., 1996, MNRAS, 281, 663

St-Louis N., Chené A.-N., Schnurr O., Nichol M.-H., 2009, ApJ, 698, 1951
St-Louis N., 2013, ApJ, 777, 9

Townsend R. H. D., Owocki S. P., 2005, MNRAS, 357, 251

ud-Doula A., Owocki S. P., 2002, ApJ, 576, 413

ud-Doula A., Owocki S. P., Townsend R. H. D., 2008, MNRAS, 385, 97

ud-Doula A., Owocki S. P., Townsend R. H. D., 2009, MNRAS, 392, 1022

ud-Doula A., Sundqvist J. O., Owocki S. P., Petit V., Townsend R. H. D.,

2013, MNRAS, 428, 2723

van Gent J. I., Lamers H. J. G. L. M., de Koter A., Morris P. W., 2001, A\&A, 372, 963

von Zeipel H., 1924, MNRAS, 84, 665

Walborn N. R., Fitzpatrick E. L., 1990, PASP, 102, 379

Weber E. J., Davis L., Jr., 1967, ApJ, 148, 217

Woosley S. E., 1993, ApJ, 405, 273

This paper has been typeset from a $\mathrm{T}_{\mathrm{E}} \mathrm{X} / \mathrm{L} \mathrm{T}_{\mathrm{E}} \mathrm{X}$ file prepared by the author. 